\title{
Predictor Based Control Strategy for Wheeled Mobile Robots Subject to Transport Delay
}

\author{
Alejandro Alvarez-Aguirre \\ Eindhoven University of Technology \\ Eindhoven, The Netherlands
}

\section{Introduction}

The performance and stability of a control system can be directly affected by a time-delay located either in its input, output, or both. In the case of a mobile robot, an input time-delay may become critical in different situations, such as when vision is used as the localization technique and a high frame per second rate is demanded, or when centralized control of multiple agents is desired, or even if very accurate regulation or tracking performance is required. It turns out that the control laws derived from the mathematical models that include an input time-delay are of a noncausal nature, thus requiring some sort of state prediction or estimation in order to implement them.

Initially, this work considers the control of a wheeled mobile robot (unicycle-type or omnidirectional) which is subject to an input time-delay. The causality problem involved in the proposed solution is tackled by considering the nonlinear case of the well known Smith predictor compensator. By doing this, the implementation of a noncausal feedback is possible and the system's performance improves under an input-time delay. It is worth noting that the necessity to consider this type of time-delay has motivated the use of discrete-time models that allow the analysis of the time-delay's effects and the synthesis of discrete-time controllers designed to compensate such effects. The complexity of the problem increases especially when the time-delay is included in the model due to the nonlinear nature of mobile robots.

Additionally, this work also proposes an extension that is able to cope with bilateral time-delays and which is based on the solution of the input time-delay problem affecting a mobile robot mentioned previously. Although rather simple, this extension is of particular importance due to the fact that it brings the proposed control strategy closer to the realm of telecontrol and teleoperation. The possibility to consider a mobile robot affected by a network time-delay rather than only an input time-delay opens a whole new range of possibilities and applications in which remotely controlling this kind of devices is possible.

In Section 2 of this chapter the classical Smith predictor is introduced together with its extensions for continuous and discrete time nonlinear systems. In the following section, the continuous time posture kinematic model of a unicycle-type and an omnidirectional mobile robot are presented. These models consider an input-time delay and are used to derive their 
exact discrete time posture kinematic models, which give way to the proposition of appropriate tracking control laws. Due to the time-delay, both control laws are found to be noncausal and thus, the requirement of a prediction scheme is concluded. The integration of the discrete time prediction scheme and the noncausal control laws is carried out in Section 4 and an extension that copes with bilateral time-delays is proposed. Numerical simulations are presented in Section 5, while conclusions and recommendations for future work are provided in Section 6.

\section{The Smith Predictor}

The Smith predictor compensator was first proposed by (Smith, 1957) and constitutes one of the simplest methods to control a Single-Input Single-Output (SISO) stable linear system with an input time-delay. Throughout the years, several modifications and extensions have been proposed to this compensator in order to accommodate, among others, Multiple-Input Multiple-Output (MIMO) linear systems, nonlinear systems, and robustness and disturbance rejection requirements. The main idea behind these Smith predictors is the use of a controller structure which extracts the time-delay out of the control loop and allows a feedback design based on a delay free system, (Michiels and Niculescu, 2007). In other words, the Smith predictor compensator enables the prediction of the states of a system at a given time instant in the future. The magnitude of the prediction time for the states is limited by the magnitude of the time-delay affecting the system. This prediction allows the implementation of a non-causal control law which can be used to control a system when it is subject to an input time-delay.

\subsection{Linear Smith Predictor}

This subsection is based on the work of (Kravaris and Wright, 1989), in which the working principle behind the Smith predictor is analyzed and where its configuration for the state space representation is explained.

Consider a linear SISO system in its state space representation and subject to an input timedelay $\tau$,

$$
\begin{aligned}
& \dot{x}(t)=A x(t)+B u(t-\tau), \\
& \dot{y}(t)=C x(t),
\end{aligned}
$$

where $x(t)$ constitutes the system's state vector of dimension $n, u(t-\tau)$ its time-delayed input, $y(t)$ its output, $A$ is an $n \times n$ constant matrix, $B$ is a constant vector of dimension $n \times 1$ and $C$ is a constant vector of dimension $1 \times n$.

The implementation of the Smith predictor for system (1) is subject to the following propositions:

Proposition 2.1 System (1) is open-loop stable.

Proposition 2.2 The delay free part of system (1), as expressed in (2), has stable zero dynamics;

$$
\begin{aligned}
& \dot{\xi}(t)=A \xi(t)+B u(t), \\
& y(t)=C \xi(t),
\end{aligned}
$$

where $\xi(t)$ is the state vector of the delay free part of the system. 


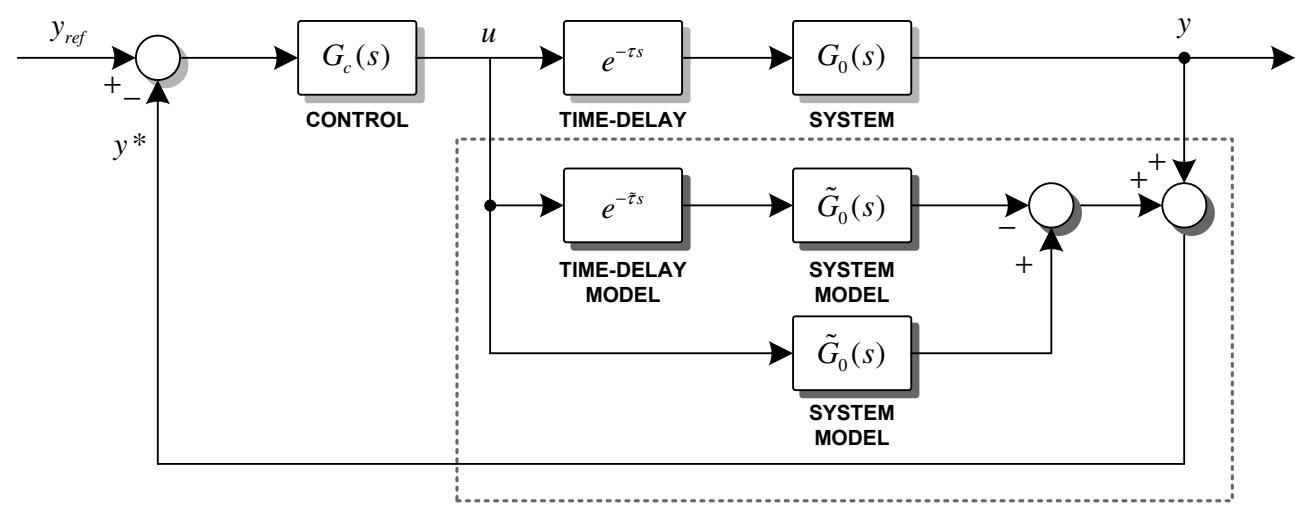

Fig. 1. Classic Smith predictor compensator block diagram.

Remark 2.3 Note that in almost every variation and extension of the Smith predictor Proposition 2.1 turns out to be fundamental and therefore can not be discarded when considering nonlinear systems. Moreover, Proposition 2.2 is also crucial for nonlinear controllers, including the input/output linearization technique, as explained in (Kravaris, 1987) and (Kravaris, 1988).

The transfer function of system (1) is given by,

$$
\frac{y(s)}{u(s)}=G_{0}(s) e^{-\tau s}
$$

where all the poles and zeros of $G_{0}(s)$ are located on the left hand-plane. The classical block diagram structure of the Smith predictor for this system is shown in Fig. 1.

In the figure, $\tilde{G}_{0}(s)$ and $\tilde{\tau}$ represent the system's and time-delay's model respectively, and $y_{\text {ref }}$ constitutes the reference signal. The main idea behind the Smith predictor is to compute the difference between the delay free and delayed model of the system. The signal that results from the predictor is added to the system's measured states in order to predict their value considering no time-delay is present. This predicted output $y^{*}$ then enters the controller $G_{\mathcal{C}}(s)$. The closed-loop transfer function resulting from the block diagram in Fig. 1 yields,

$$
\frac{y(s)}{y_{r e f}(s)}=\frac{G_{c}(s) G_{o}(s)}{1+G_{c}(s) \tilde{G}_{o}(s)+G_{c}(s)\left(G_{o}(s) e^{-\tau s}-\tilde{G}_{o}(s) e^{-\tilde{\tau} s}\right)} e^{-\tau s} .
$$

When the models of the system and the time-delay considered in the predictor are perfect, i.e. when $\tilde{G}_{o}(s)=G_{0}(s)$ and $\tilde{\tau}=\tau$, the closed-loop transfer function (4) becomes,

$$
\frac{y(s)}{y_{r e f}(s)}=\frac{G_{c}(s) G_{o}(s)}{1+G_{c}(s) G_{o}(s)} e^{-\tau s} .
$$

The structure of the closed-loop transfer function (5) and the interpretation already given to the feedback signal $y^{*}$ indicates that the parametrization of the controller $G_{c}(s)$ should be in terms of the delay free part of the model, i.e. $G_{0}(s)$. Moreover, it is worth noting that the characteristic equation of system (5) is,

$$
1+G_{c}(s) G_{o}(s)=0 .
$$




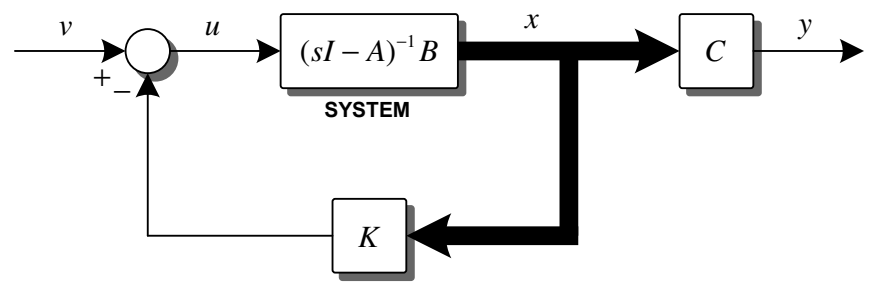

Fig. 2. Linear system in state space representation.

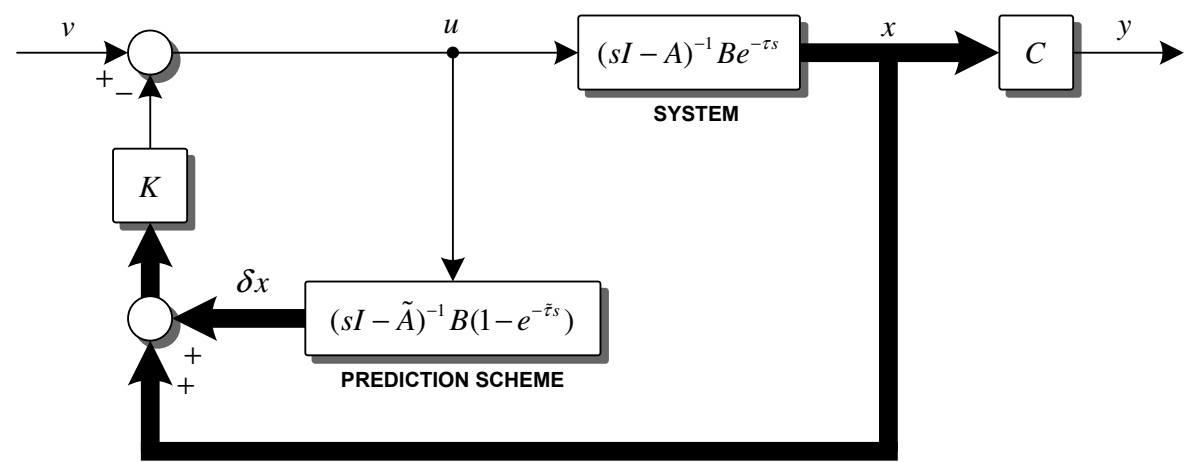

Fig. 3. Linear Smith predictor compensator in state space representation.

The concepts presented so far can be directly translated to the state space representation of a linear system (1). This, in turn, will simplify the extension of the idea behind the Smith predictor to nonlinear systems.

Consider now that the system in the state space representation is not affected by a time-delay, i.e. $\tau=0$. Moreover, the system is subject to a static feedback of the type $u(t)=v(t)-K x(t)$, where $v(t)$ is the system's reference and $K$ is a group of gains. A block representation depicting this structure is shown in Fig. 2.

The system's closed-loop transfer function is given by,

$$
\frac{y(s)}{v(s)}=\frac{C A d j(s I-A) B}{\operatorname{det}(s I-A)+K A d j(s I-A) B} .
$$

If the system is now subject to a time-delay, i.e. $\tau \neq 0$, a similar structure to the classical Smith predictor can be proposed for the state space representation. In this case, the value of the system's states if no time-delay was present can be obtained by adding a corrective signal to the states measured from the output. Such signal can be constructed by computing the difference between the delayed and the delay-free states. The prediction scheme for a system in the state space representation is depicted in Fig. 3. 
Considering once more a perfect modeling of the system and the time-delay, i.e. $\tilde{A}=A, \tilde{B}=B$ and $\tilde{\tau}=\tau$, the closed-loop transfer function of the block diagram in Fig. 3 becomes,

$$
\frac{y(s)}{v(s)}=\frac{C A d j(s I-A) B}{\operatorname{det}(s I-A)+K A d j(s I-A) B} e^{-\tau s} .
$$

It is worth noting that expressions (7) and (8) are the same except for the time-delay term $e^{-\tau s}$. It should be clear that nothing can be done with this term and that a non-causal feedback is required in order to obtain a system's response which is not time-delayed.

From the closed-loop transfer function (8) it should also be clear that this structure allows to select the closed-loop poles of the time-delayed system by using some kind of pole placement technique on the delay free part of the system. It is worth noting that (Morari, 1989) explains that if stability is the only criterion considered, the previous statement is correct. However, if the system's performance is the criterion taken into account, several considerations should be made in order to correctly tune the controller, see (Morari, 1989).

In summary, the problem of pole placement for a system subject to a time-delay can be reduced to the problem of pole placement for the delay free part of the system if a Smith-type predictor is embedded into the system's control structure.

The basic properties of the Smith predictor have been extensively explained in (Jerome and Ray, 1986) and apply to the different prediction schemes derived from it. It is worth noting that these properties assume a perfect system and time-delay model. In order to provide further insight into the behavior of the Smith predictor, these properties can be very briefly stated as:

Property 1. The Smith predictor eliminates the time-delay of the closed-loop characteristic equation of a system.

Property 2. For changes in the system's operating point, the Smith predictor provides the controller with an immediate prediction $\tau$ units of time into the future of the effects of its control action on the system's predicted output.

Property 3. The structure of the Smith predictor implicitly divides the model of the dynamical system in two parts or terms. The first one being the time-delay $e^{-\tau s}$ and the second one the remaining system dynamics $G_{0}(s)$. This two terms can then be treated in a completely independent way.

\subsection{Nonlinear Smith-Type Predictor}

This subsection is based on the work of (Kravaris and Wright, 1989), in which the Smith predictor in its state space representation is extended in order to accommodate continuous time nonlinear systems.

Consider the classic representation of a delay free, continuous time nonlinear system,

$$
\begin{aligned}
& \dot{x}(t)=f(x(t))+g(x(t)) u(t), \\
& y(t)=h(x(t)) .
\end{aligned}
$$


where $u \in \mathbb{R}^{m}$ is the system's input, $x \in \mathbb{R}^{n}$ is the system's state, and $y \in \mathbb{R}^{m}$ is the system's output.

It is assumed system (9) satisfies Propositions 2.1 and 2.2 (for a delay free system). Moreover, if the system has a relative degree vector $r_{1}, \ldots, r_{m}$ at point $x^{0}$, then following static state feedback,

$$
u(t)=\Psi(x(t), v(t))=\frac{1}{L_{g} L_{f}^{r-1} h(x(t))}\left(v(t)-L_{f}^{r} h(x(t))\right),
$$

with the auxiliary control $v(t)$ defined as,

$$
v(t)=y_{R}^{(r)}-\sum_{i=1}^{r} c_{i-1}\left(L_{f}^{(i-1)} h(x(t))-y_{R}^{(i-1)}\right),
$$

will cause the system to follow the reference $y_{R}^{(r)}$, i.e. the input/output behavior of the system satisfies the expression,

$$
y_{R}^{(r)}=v(t)
$$

In order to guarantee input/output stability, the control parameters $c_{i-1}$ should be selected in such a way that all the poles of the resulting linear subsystem are located in the left hand-plane.

Consider now a nonlinear system with an input time-delay and which satisfies Propositions 2.1 and 2.2,

$$
\begin{aligned}
& \dot{x}(t)=f(x(t))+g(x(t)) u(t-\tau), \\
& y(t)=h(x(t)) .
\end{aligned}
$$

Concerning the extension of the concept of input/output linearization for system (13), it is worth noting that, due to the fact that the system is subject to an input time-delay, it is not possible to find a causal static feedback which transforms the system into a linear delay free one by using the methodology presented so far. The best that can be done is achieve a linear input/output behavior which is time-delayed, given by,

$$
y_{R}^{(r)}=v(t-\tau)
$$

A state feedback with a predictive action similar to the Smith predictor may now be considered. In order to achieve this, the state space representation of the Smith predictor presented in Subsection 2.1 will be used.

The system's model can be used to compute a corrective signal which, when added to the measured states, predicts their values if no time-delay was present. With this, the predicted states can be fed into the controller by means of a static feedback $\Psi$. A block diagram of the resulting structure is shown in Fig. 4.

The nonlinear predictor may be characterized by the following expressions,

$$
\begin{aligned}
\dot{x}(t) & =\tilde{f}(\tilde{x}(t))+\tilde{g}(\tilde{x}(t)) u(t), \\
\dot{\hat{x}}(t) & =\tilde{f}(\hat{x}(t))+\tilde{g}(\hat{x}(t)) u(t-\tilde{\tau}), \\
\delta x(t) & =\tilde{x}(t)-\hat{x}(t),
\end{aligned}
$$




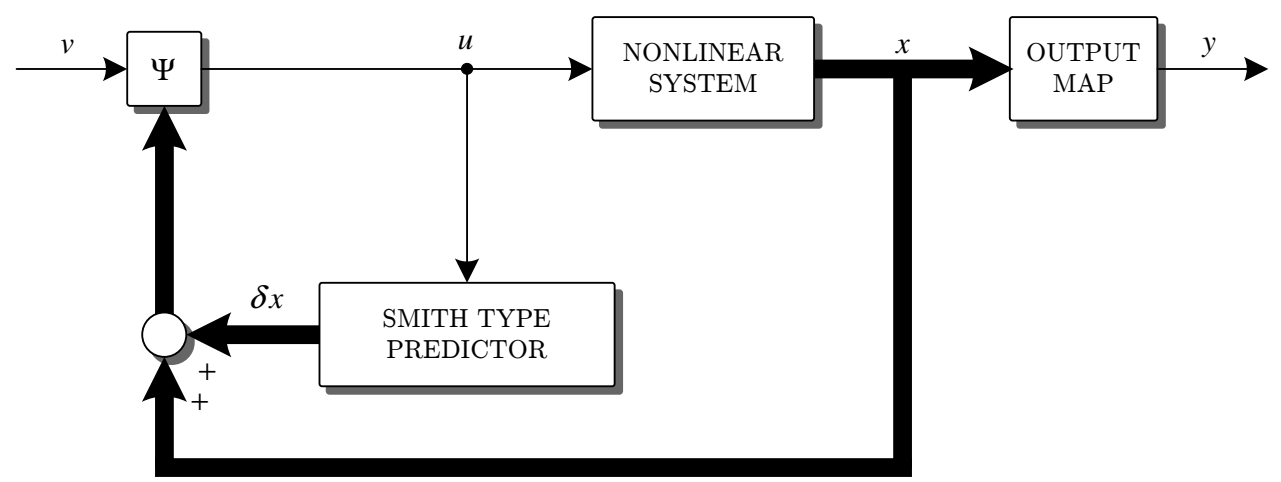

Fig. 4. Nonlinear Smith-type predictor compensator.

where $\tilde{x}(t)$ represents the states of the delay free model of the system, $\hat{x}(t)$ represents the states of the delayed model of the system, and $\delta x(t)$ represents the predictor's output, composed by computing the difference between the two models. This produces, in a similar way to the linear case, the following term entering the controller,

$$
x^{*}(t)=x(t)+\delta x(t) .
$$

A perfect modeling of the system and the time-delay, i.e $\tilde{f}=f, \tilde{g}=g, \tilde{\tau}=\tau$ and $\hat{x}(t)=x(t)$, results in $x^{*}(t)=\tilde{x}(t)$. This means that the controller is actually fed by $x^{*}(t)=\tilde{x}(t)$, which in reality is the state of the delay free model of the system and in fact constitutes the prediction of the system's state $\tau$ units of time into the future, i.e. $x(t+\tau)$.

The predicted state together with the static state feedback $\Psi$, given by equation (10), generates the following input/output behavior of the delay free model of the system,

$$
y_{R}^{(r)}=v(t)
$$

Considering this, the feedback law will now be given by,

$$
u(t)=\frac{1}{L_{g} L_{f}^{r-1} h(x(t+\tau))}\left(v(t+\tau)-L_{f}^{r} h(x(t+\tau))\right) .
$$

As the control signal (18) experiences a time-delay, the system's input becomes,

$$
u(t-\tau)=\frac{1}{L_{g} L_{f}^{r-1} h(x(t))}\left(v(t)-L_{f}^{r} h(x(t))\right),
$$

and therefore the system will track a delayed version of the reference signal.

\subsection{Discrete-Time Nonlinear Smith-Type Predictor}

The extension of the Smith predictor to discrete time nonlinear systems was carried out by (Henson and Seborg, 1994) and considers the discrete time model of a nonlinear system as,

$$
\begin{aligned}
x(k+1) & =f(x(k))+g(x(k)) u(k-\tau), \\
y(k) & =h(x(k)) .
\end{aligned}
$$


The only consideration that has to be made in order to obtain a predictor for discrete time nonlinear systems is that the modification of the Smith predictor carried out in Subsection 2.2 has to be derived in terms of the discrete time model (20).

For instance, consider that the future values of the state in model (20) are required for time instant $k+i$, i.e. $i$ time instants into the future. Such value would be given by,

$$
x(k+i)=f(x(k+i-1))+g(x(k+i-1)) u(k+i-\tau-1) .
$$

On the other hand, considering a prediction for time instant $i=\tau$, the output of the predictor presented in (15) can be expressed in discrete time based on expression (21), i.e.,

$$
\begin{aligned}
\tilde{x}(k+1) & =\tilde{f}(\tilde{x}(k))+\tilde{g}(\tilde{x}(k) u(k)), \\
\hat{x}(k+1) & =\tilde{f}(\hat{x}(k))+\tilde{g}(\hat{x}(k) u(k-\tilde{\tau})), \\
\delta x(k) & =\tilde{x}(k)-\hat{x}(k) .
\end{aligned}
$$

Once more, a perfect modeling of the system and the time-delay, i.e $\tilde{f}=f, \tilde{g}=g, \tilde{\tau}=\tau$ and $\hat{x}(k)=x(k)$, results in $x^{*}(k)=\tilde{x}(k)$. In other words, the controller is actually being fed with $x(k+\tau)$ and a noncausal control law may be implemented.

The previous results are summarized in two properties by (Henson and Seborg, 1994),

Property 1. If a perfect model of the system and the time-delay are used, the controller will receive the signal $x^{*}(k)=x(k+\tau)$ for all $k \geq 0$.

Property 2. If the closed-loop system is asymptotically stable, then $x^{*}(k)=x(k+\tau)$ in the limit as $k \rightarrow \infty$.

The cited work also explains the reasons why the proposed predictor may yield poor state predictions when mismatch between the model and the system exists or when unknown perturbations affect the system. The latter applies for both, the continuous and discrete time case.

\section{Wheeled Mobile Robots (WMR)}

A mobile robot may be defined as an electromechanical device which is capable of displacing within its workspace and can be classified according to its type of locomotion, e.g. by means of legs, wheels or tracks. A fundamental issue when considering the analysis, design, implementation and control of wheeled mobile robots (WMR) is precisely their type, layout, configuration and characteristics. For example, the wheels of a mobile robot may be conventional or omnidirectional, and of fixed or adjustable orientation. Moreover, the number, type and layout of the wheels of a WMR determines its classification and number of degrees of freedom. A practical mobile robot moving on a plane should have as minimum two degrees of freedom and as maximum three (Canudas De Wit et.al., 1996). This work features a unicycle-type mobile robot or type $(2,0)$, which possesses two degrees of mobility provided by a translational and a rotational velocity. Also included is an omnidirectional mobile robot or type $(3,0)$, which possesses three degrees of mobility provided by a rotational velocity and two linear ones. 


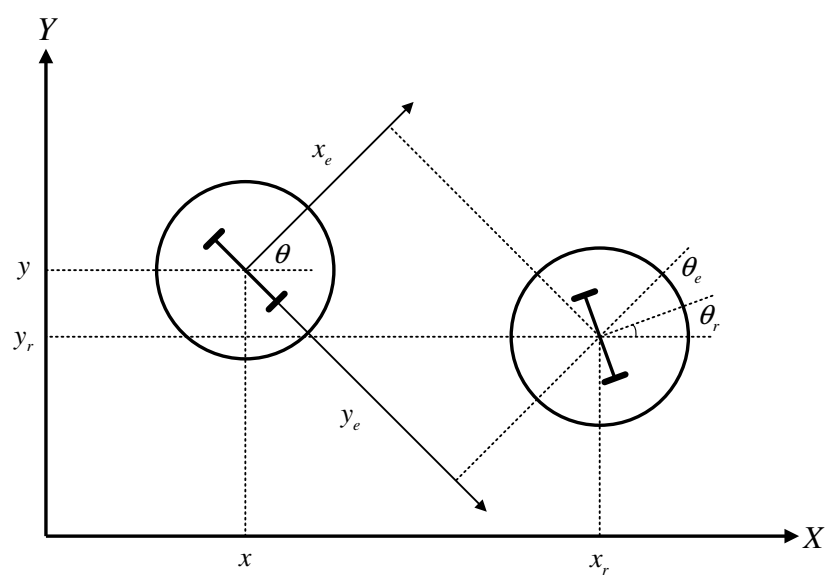

Fig. 5. Unicycle-type mobile robot and error coordinates.

\subsection{Posture Kinematic Model}

In general, the mathematical model of a WMR is nonlinear and, in cases such as the unicycletype mobile robot, may even belong to the class of systems denoted as non-holonomic, which are characterized by non-integrable restrictions in their velocities. The posture kinematic model of a mobile robot provides as output specific information about the location and orientation of the vehicle within its workspace and uses the robot's velocities as inputs. In particular, the discrete-time posture kinematic model of a mobile robot allows a closer control of the sampling time at which information is sent and received from the vehicle.

\subsubsection{Unicycle-Type Mobile Robot}

The kinematic model of a unicycle-type mobile robot can be easily derived by considering the geometric representation given in Fig. 5. The velocity components with respect to the Cartesian coordinate system $X-Y$ are obtained as in (Canudas De Wit et.al., 1996), (Campion et.al, 1996), i.e.,

$$
\begin{aligned}
& \dot{x}(t)=v(t) \cos \theta(t), \\
& \dot{y}(t)=v(t) \sin \theta(t), \\
& \dot{\theta}(t)=\omega(t),
\end{aligned}
$$

in which $x(t)$ and $y(t)$ denote the robot's position in the workspace w.r.t. the coordinate frame $X-Y, \theta(t)$ corresponds to its orientation with respect to the $X$ axis, and $v(t)$ and $\omega(t)$ represent its translational and rotational velocities respectively, which are regarded as the system's control inputs. The state vector for this robot is defined by $q(t)=\left[\begin{array}{lll}x(t) & y(t) & \theta(t)\end{array}\right]^{T}$.

When considering an implementation, the relation that exists between the system's input signals, $v(t)$ and $\omega(t)$, and the angular velocity of each wheel, $\omega_{1}(t)$ and $\omega_{2}(t)$, has been derived in Salgado (2000). Given a unicycle-type mobile robot with wheels of radius $R$ and a distance 
between the wheels and the center of the vehicle of $l$, this relation is given by,

$$
\left[\begin{array}{c}
v(t) \\
\omega(t)
\end{array}\right]=\frac{R}{2}\left[\begin{array}{cc}
1 & 1 \\
\frac{1}{l} & -\frac{1}{l}
\end{array}\right]\left[\begin{array}{c}
w_{1}(t) \\
w_{2}(t)
\end{array}\right]
$$

As explained previously, the system is subject to an input time-delay. In the case of the unicycle-type mobile robot this means that the velocities $v(t)$ and $\omega(t)$ experience an equal time-delay. The posture kinematic model of the robot subject to an input time-delay $\tau$ is derived from (23) and is given by,

$$
\begin{aligned}
& \dot{x}(t)=v(t-\tau) \cos \theta(t), \\
& \dot{y}(t)=v(t-\tau) \sin \theta(t), \\
& \dot{\theta}(t)=\omega(t-\tau),
\end{aligned}
$$

\subsubsection{Omnidirectional Mobile Robot}

The posture kinematic model of an omnidirectional mobile robot can be easily obtained by considering the geometric representation given in Fig. 6. The velocity components with respect to the axis $X-Y$ are obtained as in (Campion et.al, 1996) and (Canudas De Wit et.al., 1996),

$$
\begin{aligned}
& \dot{x}(t)=u_{1}(t) \cos \theta(t)-u_{2}(t) \cos \left(-\theta(t)+\frac{\pi}{2}\right), \\
& \dot{y}(t)=u_{1}(t) \sin \theta(t)+u_{2}(t) \sin \left(-\theta(t)+\frac{\pi}{2}\right), \\
& \dot{\theta}(t)=u_{3}(t),
\end{aligned}
$$

where point $(x(t), y(t))$ is the position of the center of the robot on the plane $X-Y$ and $\theta(t)$ is the angular position with respect to the $X$ axis. The input signals of the robot are given by $u_{1}(t), u_{2}(t)$ and $u_{3}(t)$; where $u_{3}(t)$ is given as the rotational velocity of the robot, and $u_{1}(t)$ and $u_{2}(t)$ are two orthogonal vectors, of which $u_{1}(t)$ is aligned with the reference axis of the robot. The state vector for this robot is defined by $q(t)=\left[\begin{array}{lll}x(t) & y(t) & \theta(t)\end{array}\right]^{T}$.

From Fig. 6 it also follows that the velocities of the wheels are related to the velocity components over the axes $X-Y$ and the rotational velocity by the transformation,

$$
\left[\begin{array}{l}
R \dot{\phi}_{1}(t) \\
R \dot{\phi}_{2}(t) \\
R \dot{\phi}_{3}(t)
\end{array}\right]=\left[\begin{array}{ccc}
-\sin (\theta(t)+\delta) & \cos (\theta(t)+\delta) & l \\
-\sin (\theta(t)-\delta) & -\cos (\theta(t)-\delta) & l \\
\cos \theta(t) & \sin \theta(t) & l
\end{array}\right]\left[\begin{array}{c}
\dot{x}(t) \\
\dot{y}(t) \\
\dot{\theta}(t)
\end{array}\right],
$$

where $\phi_{i}(t)$ is the angular velocity of each wheel and $R$ is its radius, $l$ denotes the distance between each wheel and the center of the vehicle and $\delta$ is the orientation of the wheel w.r.t. axes of the vehicle.

For a possible implementation, the relationship that exists between the input signals of the system $u_{1}(t), u_{2}(t)$ and $u_{3}(t)$, and the angular velocity of each wheel is given by,

$$
\left[\begin{array}{l}
R \dot{\phi}_{1}(t) \\
R \dot{\phi}_{2}(t) \\
R \dot{\phi}_{3}(t)
\end{array}\right]=\left[\begin{array}{ccc}
-\sin \delta & \cos \delta & l \\
-\sin \delta & -\cos \delta & l \\
1 & 0 & l
\end{array}\right]\left[\begin{array}{l}
u_{1}(t) \\
u_{2}(t) \\
u_{3}(t)
\end{array}\right]
$$




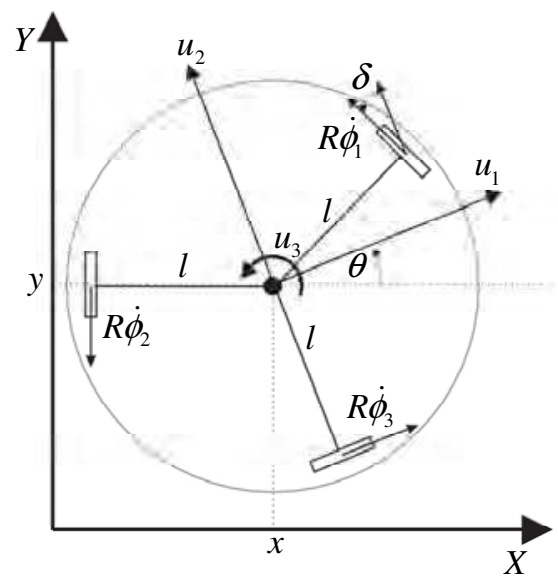

Fig. 6. Omnidirectional mobile robot.

As with the unicycle-type mobile robot, the omnidirectional mobile robot is subject to an input time-delay $\tau$, resulting in the following posture kinematic model derived from (26),

$$
\begin{aligned}
& \dot{x}(t)=u_{1}(t-\tau) \cos \theta(t)-u_{2}(t-\tau) \sin \theta(t) \\
& \dot{y}(t)=u_{1}(t-\tau) \sin \theta(t)+u_{2}(t-\tau) \cos \theta(t) \\
& \theta \dot{\theta}(t)=u_{3}(t-\tau)
\end{aligned}
$$

\subsection{Exact Discrete-Time Model}

The discretization procedure for a nonlinear system can be found in (Kotta, 1995) and consists in obtaining the solution of the system's dynamic model along the time period corresponding to the time between two sampling instants. The class of nonlinear systems considered are,

$$
\dot{x}(t)=f(x(t), u(t)) .
$$

Given a positive constant different from zero as sampling time $T$, the interval $t_{k}$ is defined as the time interval between two sampling instants in the following way:

$$
t_{k}=t \in[k T, k T+T),
$$

where: $k=0,1,2,3, \ldots$

The general solution of the differential equation that can be proposed based on system (30) at any point of the interval $t_{k}$ is given by,

$$
x(t)=x(k T)+\int_{k T}^{t} f(x(\lambda), u(\lambda)) d \lambda .
$$

In the case of sampled systems, due to their digital nature, it is generally considered that the input signals of the system are modified only during the sampling instants, which means that the system's input signal $u(t)$ in (30) is constant along the interval $t_{k}$. The value of $u(t)$ will then be that which it acquired at the beginning of the interval, i.e.,

$$
u(t)=u(k T) .
$$


The previous consideration allows rewriting equation (32), resulting in,

$$
x(t)=x(k T)+\int_{k T}^{t} f(x(\lambda), u(k T)) d \lambda .
$$

Expression (34) represents the solution of the nonlinear system given by (30) in the time instant $t$ within the time interval $t_{k}$. Consequently, if the solution presented in (34) is evaluated at the end of interval $t_{k}$, a nonlinear discrete-time model of the nonlinear system can be obtained as follows,

$$
x((k+1) T)=x(k T)+\int_{k T}^{(k+1) T} f(x(\lambda), u(k T)) d \lambda .
$$

If the integral term in (35) has an explicit solution, then the resulting function represents an exact discrete-time model given by,

$$
x((k+1) T)=x(k T)+\Phi(T, x(\lambda), u(k T)),
$$

where:

$$
\Phi(T, x(\lambda), u(k T))=\int_{k T}^{(k+1) T} f(x(\lambda), u(k T)) d \lambda .
$$

In those cases where the integral of equation (37) can not be obtained explicitly, it is possible to obtain an approximation based on the substitution of $f(x(t), u(t))$ by its Taylor series, which results in,

$$
\begin{gathered}
\Phi(T, x(\lambda), x(k T), u(k T))=\int_{k T}^{(k+1) T}\left(f(x(k T), u(k T))+(x(\lambda)-x(k T)) f^{(1)}(x(k T), u(k T))+\cdots\right. \\
\left.\cdots+\frac{(x(\lambda)-x(k T))^{n} f^{(n)}(x(k T), u(k T))}{n !}+\cdots\right) d \lambda,
\end{gathered}
$$

where,

$$
f^{(i)}(x(k T), u(k T))=\frac{\partial^{i}}{\partial x(k T)^{i}} f(x(k T), u(k T)), \quad x \in \mathbb{R}^{n}, u \in \mathbb{R}^{n} .
$$

A zero order approximation of (38) yields,

$$
\Phi(T, x(k T), u(k T))=\int_{k T}^{(k+1) T} f(x(k T), u(k T)) d \lambda=T f(x(k T), u(k T)),
$$

which results in the following approximate discrete time model,

$$
x((k+1) T)=x(k T)+T f(x(k T), u(k T)) .
$$

\subsubsection{Unicycle-Type Mobile Robot}

The procedure to obtain the discrete-time model presented in this section is explained with greater detail in Orosco (2003). Consider the continuous time posture kinematic model of a unicycle-type mobile robot as given in (23). Applying the exact discretization procedure presented in (36) results in,

$$
\left[\begin{array}{l}
x((k+1) T) \\
y((k+1) T) \\
\theta((k+1) T)
\end{array}\right]=\left[\begin{array}{l}
x(k T) \\
y(k T) \\
\theta(k T)
\end{array}\right]+\Phi(T, x(\lambda), y(\lambda), \theta(\lambda), v(k T), \omega(k T)),
$$


where,

$$
\Phi(T, x(\lambda), y(\lambda), \theta(\lambda), v(k T), \omega(k T))=\int_{k T}^{(k+1) T}\left[\begin{array}{cc}
\cos (\theta(\lambda)) & 0 \\
\sin (\theta(\lambda)) & 0 \\
0 & 1
\end{array}\right]\left[\begin{array}{c}
v(k T) \\
\omega(k T)
\end{array}\right] d \lambda
$$

As mentioned previously, the input signal $u(t)$ is considered to maintain a constant value $u(k T)$ along the interval $t_{k}$.

In order to obtain the exact discrete-time model, it is obvious that the instant value of angle $\theta(t)$ along the time interval $t_{k}$ is required. In consequence, it is necessary to obtain the solution to the differential equation proposed for this angle in (23c). Applying equation (34) for this purpose yields,

$$
\begin{aligned}
\theta(t) & \left.=\theta(k T)+\int_{k T}^{t} f(\theta(\lambda), \omega(k T))\right) d \lambda \\
& =\theta(k T)+[t-k T] \omega(k T) .
\end{aligned}
$$

The integrals proposed in (43) are solved using the value of $\theta(t)=\theta(\lambda)$ given by (44). For the first integral this results in,

$$
\begin{aligned}
\int_{k T}^{(k+1) T} v(k T) \cos (\theta(\lambda)) d \lambda & =\int_{k T}^{(k+1) T} v(k T) \cos (\theta(k T)+[\lambda-k T] \omega(k T)) d \lambda \\
& =\frac{v(k T)}{\omega(k T)}(\sin (\theta(k T)+T \omega(k T))-\sin \theta(k T)) .
\end{aligned}
$$

For the second integral the result yields,

$$
\begin{aligned}
\int_{k T}^{(k+1) T} v(k T) \sin (\theta(\lambda)) d \lambda & =\int_{k T}^{(k+1) T} v(k T) \sin (\theta(k T)+[\lambda-k T] \omega(k T)) d \lambda \\
& =-\frac{v(k T)}{\omega(k T)}(\cos (\theta(k T)+T \omega(k T))-\cos \theta(k T)) .
\end{aligned}
$$

Finally the third integral is,

$$
\begin{aligned}
\int_{k T}^{(k+1) T} \omega(k T) d \lambda & =\left.\omega(k T) \lambda\right|_{k T} ^{(k+1) T} \\
& =T \omega(k T) .
\end{aligned}
$$

Applying the sum-to-product trigonometric identity on (45) and (46) results in,

$$
\Phi(T, x(\lambda), y(\lambda), \theta(\lambda), v(k T), \omega(k T))=\left[\begin{array}{c}
2 \frac{v(k T)}{\omega(k T)} \sin \left(\frac{T \omega(k T)}{2}\right) \cos \left(\theta(k T)+\frac{T \omega(k T)}{2}\right) \\
2 \frac{v(k T)}{\omega(k T)} \sin \left(\frac{T \omega(k T)}{2}\right) \sin \left(\theta(k T)+\frac{T \omega(k T)}{2}\right) \\
T \omega(k T)
\end{array}\right] .
$$


The exact discrete-time model of a unicycle-type mobile robot is then given by,

$$
\begin{aligned}
& x((k+1) T)=x(k T)+2 v(k T) \frac{\sin \left(\frac{T}{2} \omega(k T)\right)}{\omega(k T)} \cos \left(\theta(k T)+\frac{T}{2} \omega(k T)\right), \\
& y((k+1) T)=y(k T)+2 v(k T) \frac{\sin \left(\frac{T}{2} \omega(k T)\right)}{\omega(k T)} \sin \left(\theta(k T)+\frac{T}{2} \omega(k T)\right), \\
& \theta((k+1) T)=\theta(k T)+T \omega(k T) .
\end{aligned}
$$

It is worth noting that in the model, states (49a) and (49b) become undefined in the term $\frac{\sin \left(\frac{T}{2} \omega(k T)\right)}{\omega(k T)}$ when $\omega(k T)=0$. However, by l'Hôpital's rule it is possible to approximate this term by $\frac{T}{2}$. The following function is proposed to account for this situation,

$$
\gamma(\omega(k T))=\left\{\begin{array}{ccc}
\frac{\sin \left(\frac{T}{2} \omega(k T)\right)}{\omega(k T)} & \text { if } & \omega(k T) \neq 0, \\
\frac{T}{2} & \text { if } & \omega(k T)=0 .
\end{array}\right.
$$

The discrete-time exact model of a unicycle-type mobile robot is then given by,

$$
\begin{aligned}
& x((k+1) T)=x(k T)+2 v(k T) \gamma(\omega(k T)) \cos \left(\theta(k T)+\frac{T}{2} \omega(k T)\right), \\
& y((k+1) T)=y(k T)+2 v(k T) \gamma(\omega(k T)) \sin \left(\theta(k T)+\frac{T}{2} \omega(k T)\right), \\
& \theta((k+1) T)=\theta(k T)+T \omega(k T) .
\end{aligned}
$$

In the same way as (51), the exact discrete-time model of the robot with delayed inputs is obtained based on the input delayed posture kinematic model (25). Once more assuming the input signals are constant during a sampling interval, direct integration of (25c) yields,

$$
\theta(t)=\theta(k T)+[t-k T] \omega(k T-\tau) .
$$

Substituting (52) in (25a) and (25b) and integrating them results in,

$$
\begin{aligned}
& x(t)=x(k T)+\frac{v(k T-\tau)}{\omega(k T-\tau)}(\sin (\theta+T \omega(k T-\tau))-\sin \theta), \\
& y(t)=y(k T)-\frac{v(k T-\tau)}{\omega(k T-\tau)}(\cos (\theta+T \omega(k T-\tau))-\cos \theta),
\end{aligned}
$$

while the integration of $(25 c)$ in the interval $[k T,(k+1) T]$ yields,

$$
\theta(t)=\theta(k T)+T \omega(k T-\tau) .
$$

After some algebraic and trigonometric manipulations the exact discrete time model of the unicycle-type mobile robot results in,

$$
\begin{aligned}
& x((k+1) T)=x(k T)+2 v(k T-\tau) \gamma(\omega(k T-\tau)) \cos \left(\theta(k T)+\frac{T \omega(k T-\tau)}{2}\right), \\
& y((k+1) T)=y(k T)+2 v(k T-\tau) \gamma(\omega(k T-\tau)) \sin \left(\theta(k T)+\frac{T \omega(k T-\tau)}{2}\right), \\
& \theta((k+1) T)=\theta(k T)+T \omega(k T-\tau),
\end{aligned}
$$


where function $\gamma(\omega(k T-\tau))$ satisfies,

$$
\gamma(\omega(k T-\tau))=\left\{\begin{array}{ccc}
\frac{\sin \left(\frac{T}{2} \omega(k T-\tau)\right)}{\omega(k T-\tau)} & \text { if } & \omega(k T-\tau) \neq 0, \\
\frac{T}{2} & \text { if } & \omega(k T-\tau)=0 .
\end{array}\right.
$$

For simplification purposes, the following notation will be adopted,

$$
\zeta=\zeta(k T), \zeta^{ \pm}=\zeta(k T \pm T), \zeta^{[ \pm n]}=\zeta(k T \pm n T) .
$$

Considering the notation change proposed in (57), the exact discrete-time posture kinematic model of the unicycle-type mobile robot can be expressed as,

$$
\begin{aligned}
& x^{+}=x+2 v^{-\tau} \gamma\left(\omega^{-\tau}\right) \cos \left(\theta+\frac{T \omega^{-\tau}}{2}\right), \\
& y^{+}=y+2 v^{-\tau} \gamma\left(\omega^{+}\right) \sin \left(\theta+\frac{T \omega^{-\tau}}{2}\right), \\
& \theta^{+}=\theta+T \omega^{-\tau},
\end{aligned}
$$

where function $\gamma\left(\omega^{-\tau}\right)$ satisfies,

$$
\gamma\left(\omega^{-\tau}\right)=\left\{\begin{array}{ccc}
\frac{\sin \left(\frac{T}{2} \omega^{-\tau}\right)}{\omega^{-\tau}} & \text { if } \quad \omega^{-\tau} \neq 0 \\
\frac{T}{2} & \text { if } \quad \omega^{-\tau}=0
\end{array}\right.
$$

\subsubsection{Omnidirectional Mobile Robot}

The exact discrete time model of the omnidirectional mobile robot subject to an input timedelay may be easily obtained by direct integration of the equations given in (29). In this sense, notice that under the assumption that the control signals are constant between sampling instances, equation $(29 \mathrm{c})$ produces,

$$
\theta(t)=\theta(k T)+[t-k T] u_{3}(k T-\tau) .
$$

Substituting (60) into (29a) and (29b) and integrating as in (37) yields,

$$
\begin{aligned}
x(t) & =x(k T)+\frac{u_{1}(k T-\tau)}{u_{3}(k T-\tau)}\left(\sin \left(\theta(t)+T u_{3}(k T-\tau)\right)-\sin \theta(t)\right) \\
& +\frac{u_{2}(k T-\tau)}{u_{3}(k T-\tau)}\left(\cos \left(\theta(t)+T u_{3}(k T-\tau)\right)-\cos \theta(t)\right) \\
y(t) & =y(k T)-\frac{u_{1}(k T-\tau)}{u_{3}(k T-\tau)}\left(\cos \left(\theta(t)+T u_{3}(k T-\tau)\right)-\cos \theta(t)\right) \\
& +\frac{u_{2}(k T-\tau)}{u_{3}(k T-\tau)}\left(\sin \left(\theta(t)+T u_{3}(k T-\tau)\right)-\sin \theta(t)\right) .
\end{aligned}
$$


After some algebraic and trigonometric manipulations the exact discrete time model of the omnidirectional mobile robot results in,

$$
\begin{aligned}
& x^{+}=x+2 u_{1}^{-\tau} \gamma\left(u_{3}^{-\tau}\right) \cos \left(\theta+\frac{T u_{3}^{-\tau}}{2}\right)-2 u_{2}^{-\tau} \gamma\left(u_{3}^{-\tau}\right) \sin \left(\theta+\frac{T u_{3}^{-\tau}}{2}\right), \\
& y^{+}=y+2 u_{1}^{-\tau} \gamma\left(u_{3}^{-\tau}\right) \sin \left(\theta+\frac{T u_{3}^{-\tau}}{2}\right)+2 u_{2}^{-\tau} \gamma\left(u_{3}^{-\tau}\right) \cos \left(\theta+\frac{T u_{3}^{-\tau}}{2}\right), \\
& \theta^{+}=\theta_{0}+u_{3}^{-\tau} .
\end{aligned}
$$

where the function $\gamma\left(u_{3}^{-\tau}\right)$ accounts for terms that become undefined (as with the unicycletype mobile robot) and satisfies,

$$
\gamma\left(u_{3}^{-\tau}\right)=\left\{\begin{array}{cc}
\frac{\sin \left(\frac{T}{2} u_{3}^{-\tau}\right)}{u_{3}^{-\tau}} & \text { if } u_{3}^{-\tau} \neq 0, \\
\frac{T}{2} & \text { if } u_{3}^{-\tau}=0 .
\end{array}\right.
$$

\subsection{Tracking Controller}

Tracking a trajectory constitutes one of the simplest tasks a mobile robot can perform and constitutes the basis for achieving more complex behaviors. Designing a tracking controller based on a feedback linearization for a unicycle-type mobile robot is not straightforward due to its non-holonomic constraints, as reported by (Brockett, 1983). On the other hand, in the case of the omnidirectional mobile robot, a tracking controller by means of a full state feedback is possible, constituting the best option for this type of robot.

\subsubsection{Unicycle-Type Mobile Robot}

According to Lefeber et.al (2001), the problem of path-tracking for a unicycle-type mobile robot can be stated as the requirement for the robot to follow a reference trajectory with state $q_{r}(t)=\left[\begin{array}{lll}x_{r}(t) & y_{r}(t) & \theta_{r}(t)\end{array}\right]^{T}$ generated by an exosystem with kinematics,

$$
\begin{aligned}
& \dot{x}_{r}(t)=v_{r}(t) \cos \theta_{r}(t), \\
& \dot{y}_{r}(t)=v_{r}(t) \sin \theta_{r}(t), \\
& \dot{\theta}_{r}(t)=\omega_{r}(t),
\end{aligned}
$$

where $v_{r}(t)$ and $\omega_{r}(t)$ are continuous functions of time given as the reference velocities by,

$$
\begin{aligned}
v_{r}(t) & =\sqrt{\dot{x}_{r}^{2}(t)+\dot{y}_{r}^{2}(t)}, \\
\omega_{r}(t) & =\frac{\dot{x}_{r}(t) \ddot{y}_{r}(t)-\ddot{x}_{r}(t) \dot{y}_{r}(t)}{\dot{x}_{r}^{2}(t)+\dot{y}_{r}^{2}(t)} .
\end{aligned}
$$

From Fig. 5, it follows that the position errors between the mobile robot and the reference system can be expressed in terms of the error coordinates $q_{e}(t)=\left[\begin{array}{lll}x_{e}(t) & y_{e}(t) & \theta_{e}(t)\end{array}\right]^{T}$ on a moving coordinate frame mounted on the robot, i.e.

$$
\left[\begin{array}{l}
x_{e}(t) \\
y_{e}(t) \\
\theta_{e}(t)
\end{array}\right]=\left[\begin{array}{ccc}
\cos \theta(t) & \sin \theta(t) & 0 \\
-\sin \theta(t) & \cos \theta(t) & 0 \\
0 & 0 & 1
\end{array}\right]\left[\begin{array}{l}
x_{r}(t)-x(t) \\
y_{r}(t)-y(t) \\
\theta_{r}(t)-\theta(t)
\end{array}\right],
$$


which yield the following error dynamics when derived w.r.t to time,

$$
\begin{aligned}
& \dot{x}_{e}(t)=\omega(t) y_{e}(t)-v(t)+v_{r}(t) \cos \theta_{e}(t), \\
& \dot{y}_{e}(t)=-\omega(t) x_{e}(t)+v_{r}(t) \sin \theta_{e}(t), \\
& \dot{\theta}_{e}(t)=\omega_{r}(t)-\omega(t) .
\end{aligned}
$$

Since the error dynamics are also affected by the time-delay they are actually expressed as,

$$
\begin{aligned}
& \dot{x}_{e}(t)=\omega(t-\tau) y_{e}(t)-v(t-\tau)+v_{r}(t) \cos \theta_{e}(t), \\
& \dot{y}_{e}(t)=-\omega(t-\tau) x_{e}(t)+v_{r}(t) \sin \theta_{e}(t), \\
& \dot{\theta}_{e}(t)=\omega_{r}(t)-\omega(t-\tau) .
\end{aligned}
$$

Due to the fact that the delayed error dynamics (68) can not be integrated explicitly, their approximate Euler-discretization is computed, and results in,

$$
\begin{aligned}
& x_{e}^{+}=x_{e}+T\left(\omega^{-\tau} y_{e}-v^{-\tau}+v_{r} \cos \theta_{e}\right), \\
& y_{e}^{+}=y_{e}+T\left(-\omega^{-\tau} x_{e}+v_{r} \sin \theta_{e}\right), \\
& \theta_{e}^{+}=\theta_{e}+T\left(\omega_{r}-\omega^{-\tau}\right) .
\end{aligned}
$$

In the continuous time case, the approach presented in (Lefeber et.al, 2001) proposes the use of a cascaded structure based on the error dynamics (67) that results in the use of linear control laws. The same idea has been used in (Nešić and Loría, 2004) for the discrete time case, in which the following controller has been proposed,

$$
\begin{aligned}
\omega^{-\tau} & =\omega_{r}+c_{1} \theta_{e}, \\
v^{-\tau} & =v_{r}+c_{2} x_{e}+T \vartheta,
\end{aligned}
$$

where $c_{1}, c_{2}, v_{r}$ and $\omega_{r}$ are the same as in the continuous-time control law proposed in Lefeber et.al (2001). The term $T \vartheta$ is an extra control input should be designed to improve the performance of the system.

Considering a correcting term given by $\vartheta=-\frac{c_{3} \omega_{r} y_{e}}{T}$, where $c_{3}$ is a gain, and modifying (70a) in order to accommodate more aggressive angle variations, the following controller is proposed,

$$
\begin{aligned}
\omega^{-\tau} & =\omega_{r}+c_{1} \sin \theta_{e}, \\
v^{-\tau} & =v_{r}+c_{2} x_{e}-c_{3} \omega_{r} y_{e} .
\end{aligned}
$$

It is worth noting that a practical feedback should be synthesized at time $t$ or, more precisely, at time instant $k T$. In this case, feedback (71) results in an anticipative controller given by,

$$
\begin{aligned}
\omega & =\omega_{r}^{+\tau}+c_{1} \sin \theta_{e}^{+\tau}, \\
v & =v_{r}^{+\tau}+c_{2} x_{e}^{+\tau}-c_{3} \omega_{r}^{+\tau} y_{e}^{+\tau},
\end{aligned}
$$

which yields the anticipated error coordinates,

$$
\left[\begin{array}{l}
x_{e}^{+\tau} \\
y_{e}^{+\tau} \\
\theta_{e}^{+\tau}
\end{array}\right]=\left[\begin{array}{ccc}
\cos \theta^{+\tau} & \sin \theta^{+\tau} & 0 \\
-\sin \theta^{+\tau} & \cos \theta^{+\tau} & 0 \\
0 & 0 & 1
\end{array}\right]\left[\begin{array}{l}
x_{r}^{+\tau}-x^{+\tau} \\
y_{r}^{+\tau}-y^{+\tau} \\
\theta_{r}^{+\tau}-\theta^{+\tau}
\end{array}\right] .
$$


Remark 3.1 The control law proposed in (72) with the error coordinates (73) are non-causal expressions which require the state values $\tau$ samples of time into the future. The Smith-like prediction strategy proposed in Section 2 will be used to generate these values.

\subsubsection{Omnidirectional Mobile Robot}

Given the discrete-time input delayed posture kinematic model of the omnidirectional robot (62), the following output is proposed in order to obtain a fully linearizing feedback controller,

$$
y=h(q)=\left[\begin{array}{l}
h_{1}(q) \\
h_{2}(q) \\
h_{3}(q)
\end{array}\right]=\left[\begin{array}{l}
x \\
y \\
\theta
\end{array}\right] .
$$

Since the relative degree of system (62) w.r.t the proposed inputs in (74) is 3, a fully linearizing feedback controller may be implemented. Deriving the output once yields,

$$
\left[\begin{array}{l}
h_{1}^{+}\left(q, u^{-\tau}\right) \\
h_{2}^{+}\left(q, u^{-\tau}\right) \\
h_{3}^{+}\left(q, u^{-\tau}\right)
\end{array}\right]=\left[\begin{array}{c}
x+2 u_{1}^{-\tau} \gamma\left(u_{3}^{-\tau}\right) \cos \left(\theta+\frac{T u_{3}^{-\tau}}{2}\right)-2 u_{2}^{-\tau} \gamma\left(u_{3}^{-\tau}\right) \sin \left(\theta+\frac{T u_{3}^{-\tau}}{2}\right) \\
y+2 u_{1}^{-\tau} \gamma\left(u_{3}^{-\tau}\right) \sin \left(\theta+\frac{T u_{3}^{-\tau}}{2}\right)+2 u_{2}^{-\tau} \gamma\left(u_{3}^{-\tau}\right) \cos \left(\theta+\frac{T u_{3}^{-\tau}}{2}\right) \\
\theta_{0}+T u_{3}^{-\tau}
\end{array}\right] .
$$

In order to obtain a controller that fully decouples the system, a set of auxiliary controllers $v_{1}$, $v_{2} \mathrm{y} v_{3}$ are proposed such that the closed-loop system satisfies,

$$
\left[\begin{array}{l}
h_{1}^{+}\left(q, u^{-\tau}\right) \\
h_{2}^{+}\left(q, u^{-\tau}\right) \\
h_{3}^{+}\left(q, u^{-\tau}\right)
\end{array}\right]=\left[\begin{array}{l}
v_{1} \\
v_{2} \\
v_{3}
\end{array}\right] .
$$

The trajectory tracking problem is solved by proposing,

$$
\begin{aligned}
& v_{1}=x_{r}^{+}-k_{1} e_{1}, \\
& v_{2}=y_{r}^{+}-k_{2} e_{2}, \\
& v_{3}=\theta_{r}^{+}-k_{3} e_{3},
\end{aligned}
$$

with:

$$
\begin{aligned}
& e_{x}=x-x_{r}, \\
& e_{y}=y-y_{r}, \\
& e_{\theta}=\theta-\theta_{r},
\end{aligned}
$$

where $q_{r}=\left[\begin{array}{lll}x_{r} & y_{r} & \theta_{r}\end{array}\right]^{T}$ constitute the robot's desired trajectories, $q_{e}=\left[\begin{array}{lll}e_{x} & e_{y} & e_{\theta}\end{array}\right]^{T}$ denote the tracking positions errors and $K=\left[\begin{array}{lll}k_{1} & k_{2} & k_{3}\end{array}\right]^{T}$ are the gains of the auxiliary controllers.

Using (75) and (76), a fully linearizing controller is given by,

$$
\left[\begin{array}{l}
u_{1}^{-\tau} \\
u_{2}^{-\tau} \\
u_{3}^{-\tau}
\end{array}\right]=\left[\begin{array}{c}
\frac{\left[v_{1}-x\right] \cos \left(\theta+\frac{T u_{3}^{-\tau}}{2}\right)+\left[v_{2}-y\right] \sin \left(\theta+\frac{T u_{3}^{-\tau}}{2}\right)}{2 \gamma\left(u_{3}^{-\tau}\right)} \\
\frac{\left[v_{2}-y\right] \cos \left(\theta+\frac{T u_{3}^{-\tau}}{2}\right)-\left[v_{1}-x\right] \sin \left(\theta+\frac{T u_{3}^{-\tau}}{2}\right)}{2 \gamma\left(u_{3}^{-\tau}\right)} \\
\frac{v_{3}-\theta}{T}
\end{array}\right] .
$$


It is possible to obtain the controllers $u_{1}, u_{2}$ and $u_{3}$ in the current time instant by shifting expression (79) $\tau$ units of time, i.e.,

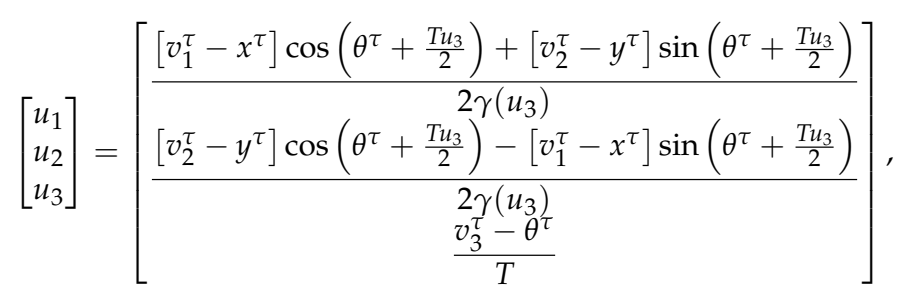

where,

$$
\begin{aligned}
& v_{1}^{\tau}=x_{r}^{\tau+1}-k_{1} e_{1}^{\tau}, \\
& v_{2}^{\tau}=y_{r}^{\tau+1}-k_{2} e_{2}^{\tau}, \\
& v_{3}^{\tau}=\theta_{r}^{\tau+1}-k_{3} e_{3}^{\tau},
\end{aligned}
$$

with:

$$
\begin{aligned}
& e_{1}^{\tau}=x^{\tau}-x_{r}^{\tau}, \\
& e_{2}^{\tau}=y^{\tau}-y_{r}^{\tau}, \\
& e_{3}^{\tau}=\theta^{\tau}-\theta_{r}^{\tau} .
\end{aligned}
$$

Remark 3.2 Equivalent to Remark 3.1, the control law proposed in (80)-(82) is non-causal and will therefore require a prediction strategy such as the one presented in Section 2 in order to obtain the state values $\tau$ samples of time into the future.

Remark 3.3 Note that the control law for the unicycle-type mobile robot (72) with the error coordinates (73) and for the omnidirectional robot (80)-(82) both require the values of the desired trajectory $\tau$ and $\tau+1$ samples of time into the future. If these values could be provided, the mobile robot would track the desired trajectories in the current time instant, i.e. $q_{r}=\left[\begin{array}{lll}x_{r} & y_{r} & \theta_{r}\end{array}\right]^{T}$. Although ideal, knowing the reference trajectory a priori is not possible in most cases. Consequently this constraint can be relaxed by making use of the desired trajectories in the current time instant $k T$ and $(k+1) T$. The result will be that the mobile robot will track a delayed version of the desired trajectories, i.e., $q_{r}^{-\tau}=\left[\begin{array}{llll}x_{r}^{-\tau} & y_{r}^{-\tau} & \theta_{r}^{-\tau}\end{array}\right]^{T}$. Simulation results in Section 5 will further clarify this point.

\section{Time-Delay Compensation}

The prediction strategy and noncausal control laws of the previous sections are seamlessly integrated to compensate for an input time-delay in a mobile robot. Moreover, a simple extension to the nonlinear predictor's structure is proposed in order to cope with bilateral timedelays, moving towards implementing a fully telecontrolled system. 


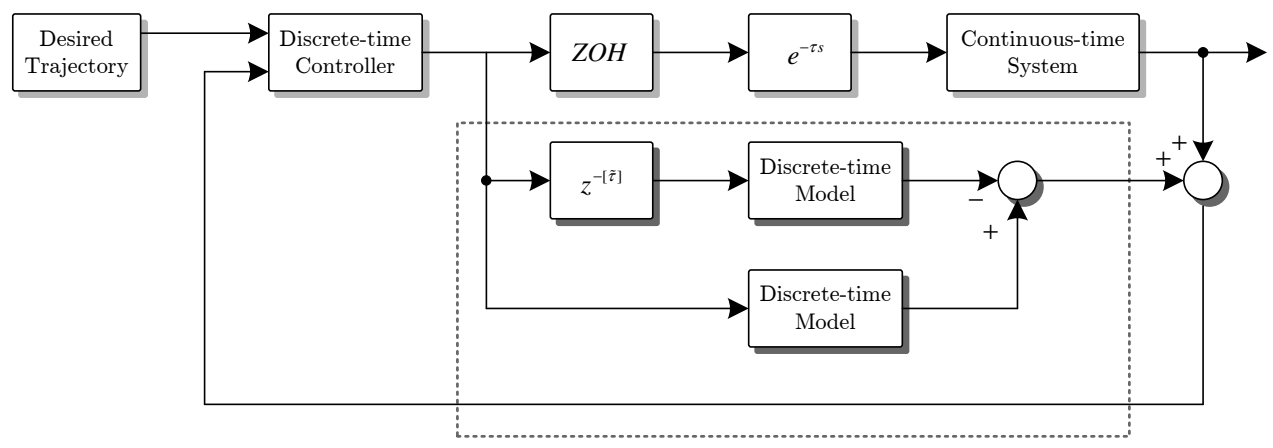

Fig. 7. Input time-delay compensation.

\subsection{Input Time-Delay Compensation}

The discrete-time nonlinear Smith-like predictor presented in Section 2 can be used to implement the noncausal control laws obtained in Section 3 for either the unicycle-type or the omnidirectional mobile robot in order to compensate the presence of an input time-delay. The integration of both schemes is straightforward and shown in Fig. 7.

The block denoted as Reference Trajectory provides the reference signals $q_{r}, v_{r}$ and $\omega_{r}$ for the mobile robots. As noted in Remark 3.3, the most general assumption is that the reference trajectory will be provided w.r.t to the current sampling instant and therefore the mobile robot will track a delayed version of this signal. The noncausal control law is computed in the Discrete Control block and requires as input the predicted states of the system and the reference trajectory. The Smith-like predictor is implemented in the blocks within the dotted line and contains the time-delay's model and the delayed and delay free models of the system. The blocks representing the physical robot and the time-delay affecting its input are also shown in the figure.

\subsection{Bilateral Time-Delay Compensation}

When studying the behavior of the predictor, the controller and the mobile robot for the input time-delay case, several observations can be made. Due to the structure of the predictor, one might say that it acts as a kind of full state observer, where it looks to reproduce the system's behavior without a time-delay. Due to the fact that the controller receives the predictor's output, which is not experiencing an input time-delay, it is able to compute a control law which will track the reference trajectory in the current time instant. It then follows that, if the control signal is delayed, the system receiving it will track a delayed version of the reference trajectory.

When considering a bidirectional time-delay, a delay in the system's outputs is also present. In this case the system is subject to a forward $\tau_{f}$ and backward $\tau_{b}$ time-delay, as explained in (Hokayem and Spong, 2006). If the Smith-like predictor as presented previously is applied to this case, the robot's performance is obviously degraded. The reason for this is, of course, the output time-delay affecting the robot. What happens is that the comparison carried out between the delayed outputs of the system and the delayed model is no longer relevant due 
to the fact that the model is not accounting for the output time-delay. As this happens, the predictor starts feeding the controller with information that is not relevant, which in turn, produces control outputs that are not adequate. This inadequate outputs are then received by the system after a time-delay and a "vicious" cycle is started.

It appears that the problem in the bidirectional delay case when considering the Smith-like predictor is that the correcting term in the predictor is not bringing the predictor's states closer to the system's states. If this term was able to draw the systems closer, then the predictor would be able to start providing the controller with the correct information and thus, the robot's performance would improve.

Assume for the time being that the forward and backward time-delays are equal and constant, i.e. $\tau_{b}=\tau_{f}=\tau$. Assume also that the time-delays in the communication channel are modeled perfectly, i.e. $\tilde{\tau}=\tau$. Note how the backward time-delay does not affect the robot's states, yielding the usual input delayed continuous time posture kinematic model for the unicycle-type (25) and omnidirectional (29) mobile robots.

Nevertheless, once the robot's outputs travel back to the controller side they are affected by the backward time-delay, resulting in,

$$
q(t-\tau)=\left[\begin{array}{l}
x(t-\tau) \\
y(t-\tau) \\
\theta(t-\tau)
\end{array}\right]
$$

Considering (83), a simple but logical solution to produce a relevant comparison within the predictor would be to also delay the outputs of the delayed model in the predictor by $\tau_{b}=$ $\tau$. The modified continuous time nonlinear predictor may be characterized by the following expressions,

$$
\begin{aligned}
\dot{\tilde{x}}(t) & =\tilde{f}(\tilde{x}(t))+\tilde{g}(\tilde{x}(t)) u(t), \\
\dot{\hat{x}}(t) & =\tilde{f}(\hat{x}(t))+\tilde{g}(\hat{x}(t)) u(t-\tau), \\
\delta x(t) & =\tilde{x}(t)-\hat{x}(t-\tau),
\end{aligned}
$$

whereas the discrete time version is given by,

$$
\begin{aligned}
\tilde{x}(k+1) & =\tilde{f}(\tilde{x}(k))+\tilde{g}(\tilde{x}(k) u(k)), \\
\hat{x}(k+1) & =\tilde{f}(\hat{x}(k))+\tilde{g}(\hat{x}(k) u(k-\tau)), \\
\delta x(k) & =\tilde{x}(k)-\hat{x}(k-\tau) .
\end{aligned}
$$

Considering (84) and (85), the term entering the controller will be given by $x^{*}(t)=\tilde{x}(t)$ in the continuous time case and $x^{*}(k)=\tilde{x}(k)$ in the discrete time case respectively. Given the new correcting term the comparison carried out within the predictor is once more able to bring the predictor's states closer to the system's states and thus, the controller is fed with the "correct" outputs by the predictor. This would mean in turn that the robot will be provided by the controller with the adequate input signals to track a delayed version of the reference signal. The block diagram of the modified bilateral predictor is depicted in Figure 8 . 


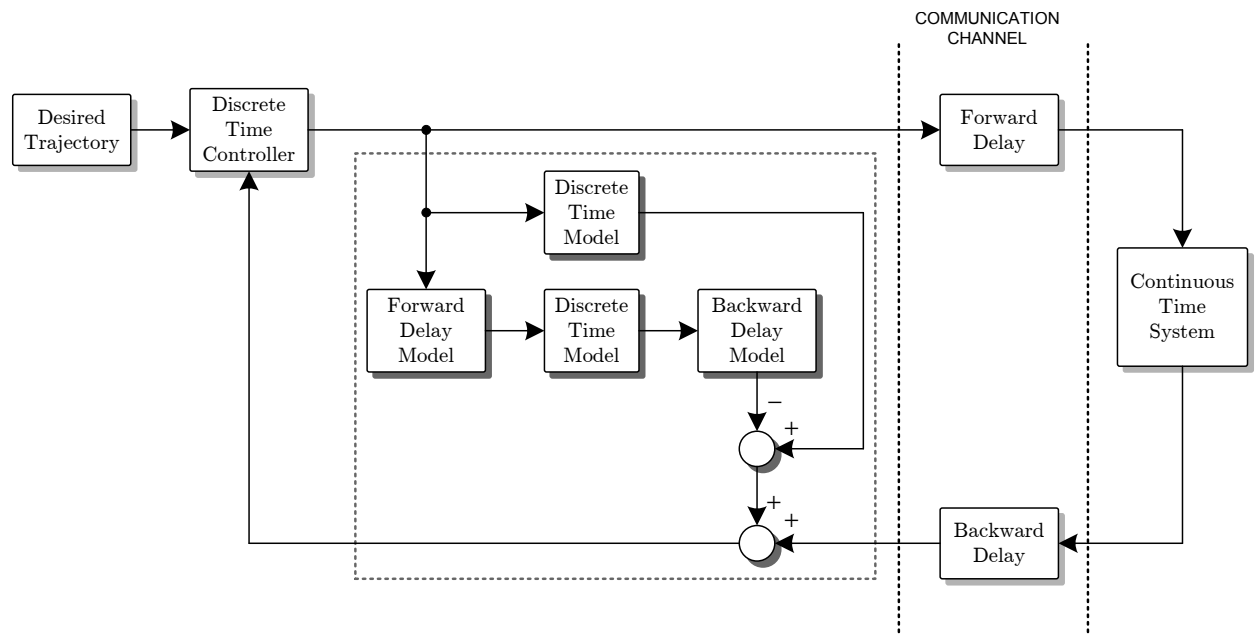

Fig. 8. Bilateral prediction strategy.

\section{Simulation Results}

This section includes numerous simulations in order to illustrate the performance of the bilateral time-delay compensation control scheme proposed in Section 4.

\subsection{Unicycle-type Mobile Robot}

For the unicycle-type mobile robot, in the first simulation the reference trajectory is a lemniscate with half length and width of $2 \mathrm{~m}$, centered at $[0,0] \mathrm{m}$ and with a desired tracking angular velocity of $0.2 \mathrm{~m} / \mathrm{s}$. The reference trajectory in the second simulation is given by a sinusoid originating at $[0.5,0.5] \mathrm{m}$, with an amplitude of $1.5 \mathrm{~m}$, an angular frequency of $0.5 \mathrm{rad} / \mathrm{s}$, a linear velocity factor of $0.1 \mathrm{~m} / \mathrm{s}$ and oriented at $\pi / 4 \mathrm{rad}$. The initial condition for the mobile robot is $q(0)=\left[\begin{array}{lll}0.1 & 0.1 & 0.0\end{array}\right]^{T}$ in the first simulation and $q(0)=\left[\begin{array}{lll}-0.3 & 1.0 & 0.0\end{array}\right]^{T}$ in the second simulation. The system's models are assumed to be perfect and are initialized with $\tilde{q}(0)=\hat{q}(0)=\left[\begin{array}{lll}0.0 & 0.0 & 0.0\end{array}\right]^{T}$ in both simulations. The controller gains in both cases are set to $c_{1}=c_{2}=c_{3}=3.0$, and the forward and backward time-delays induced by the communication channel, which are assumed to be perfectly modeled, are given by $\tau_{f}=\tau_{b}=0.25 \mathrm{sec}$. Both simulations are sampled every $T=0.05 \mathrm{sec}(20 \mathrm{~Hz})$ and are carried out during $50 \mathrm{sec}$.

The behavior of the mobile robot in the $X-Y$ plane (red) and its desired trajectory (blue) are depicted in Fig. 9 for both simulations. The small cross markers denote the simulations' initial state and the circular markers their intermediate $(25 \mathrm{sec})$ and final state (50sec). Due to the time-delay, the mobile robot lags its desired trajectory. The lag distance is determined by the time-delay's magnitude and the characteristics of the reference trajectory.

The first and third columns of Fig. 10 depict the mobile robot's states (red) and their reference (blue) for both simulations, while their errors are shown in the second and fourth columns of the same figure. The errors are computed w.r.t to a version of the reference trajectory that is delayed by $\tau$, so that both the states and the reference are in the same time base. From the 

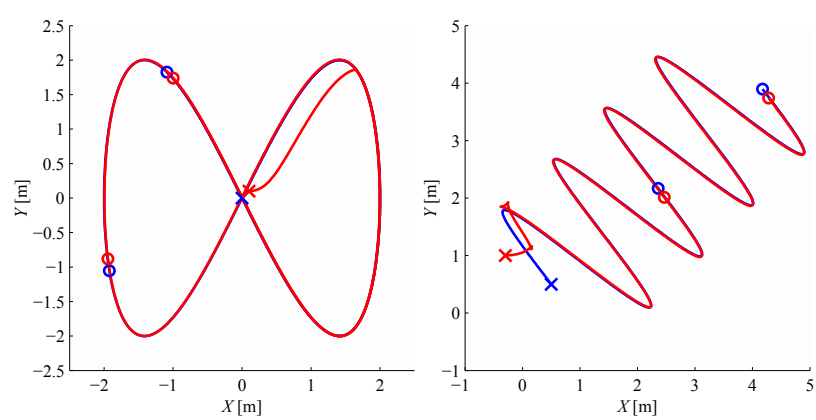

Fig. 9. Unicycle-type mobile robot: behavior in the $X-Y$ workspace.
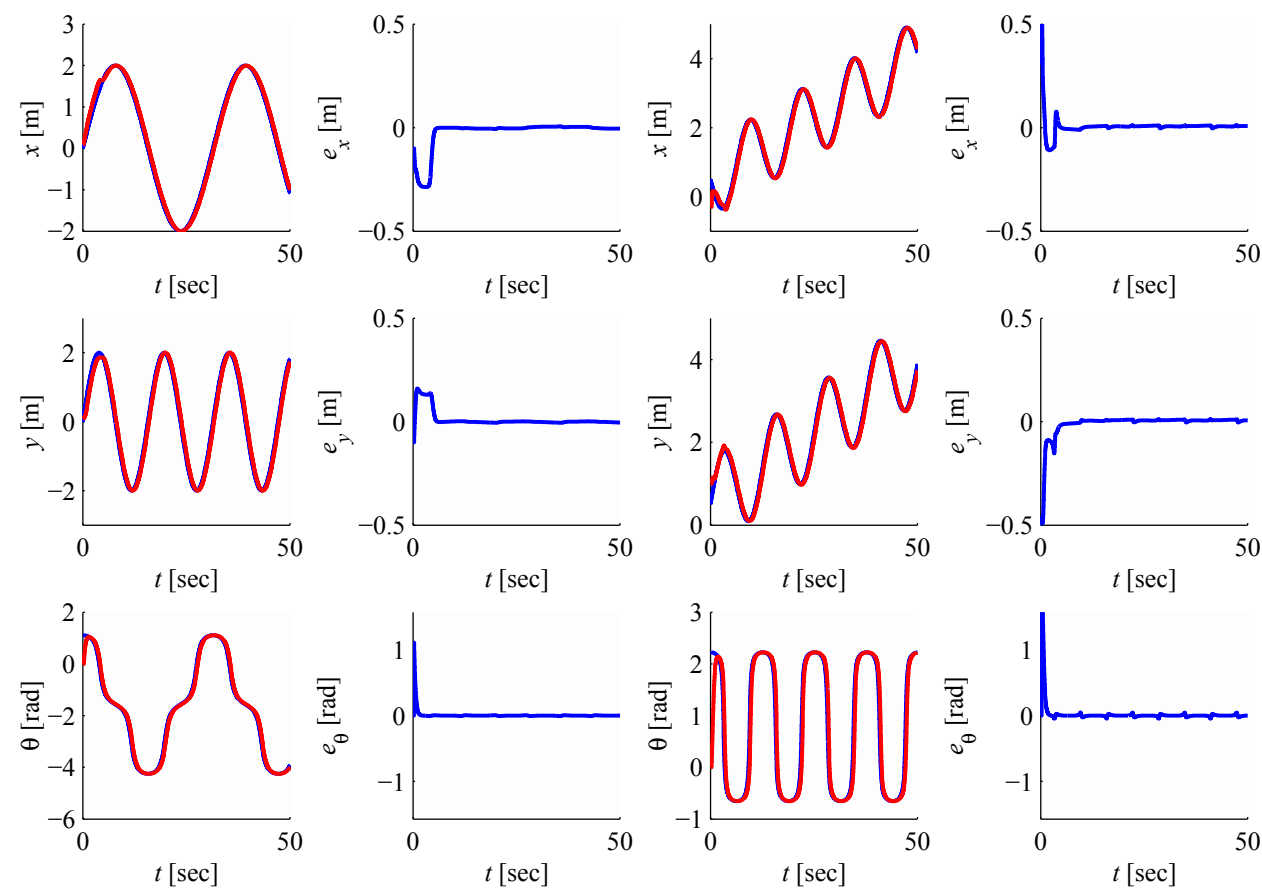

Fig. 10. Unicycle-type mobile robot: states and errors.

state plots a slight displacement between the reference trajectory and the states can be noticed due to the time-delay. This displacement is not very clear since the simulation time is too large compared with the time-delay. Given the fact that the error plots converge or practically converge to zero, it can be concluded that the unicycle-type mobile robot tracks its reference trajectory after a time $\tau$. 


\subsection{Omnidirectional Mobile Robot}

The reference trajectories and simulation conditions for the omnidirectional mobile robot are exactly the same as for the unicycle-type mobile robot, with the exception that the desired orientation is provided independently at a fixed value of $\pi / 2 \mathrm{rad}$. This is due to the fact that this type of robot does not have non-holonomic constraints and thus can follow any trajectory with an arbitrary orientation. The initial condition for the mobile robot is $q(0)=\left[\begin{array}{lll}1.0 & 1.0 & 0.0\end{array}\right]^{T}$ in the first simulation and $q(0)=\left[\begin{array}{lll}2.0 & -2.0 & 0.0\end{array}\right]^{T}$ in the second simulation. The controller gains in both cases are set to $k_{1}=-0.85, k_{2}=-0.85, k_{3}=0.3$.
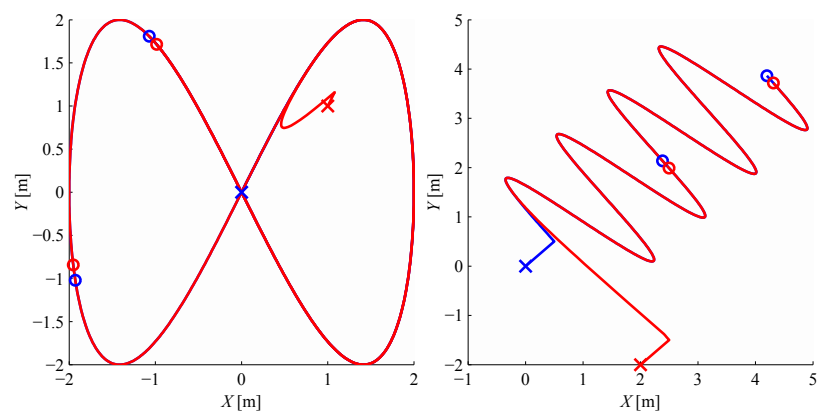

Fig. 11. Omnidirectional mobile robot in its workspace $X-Y$.
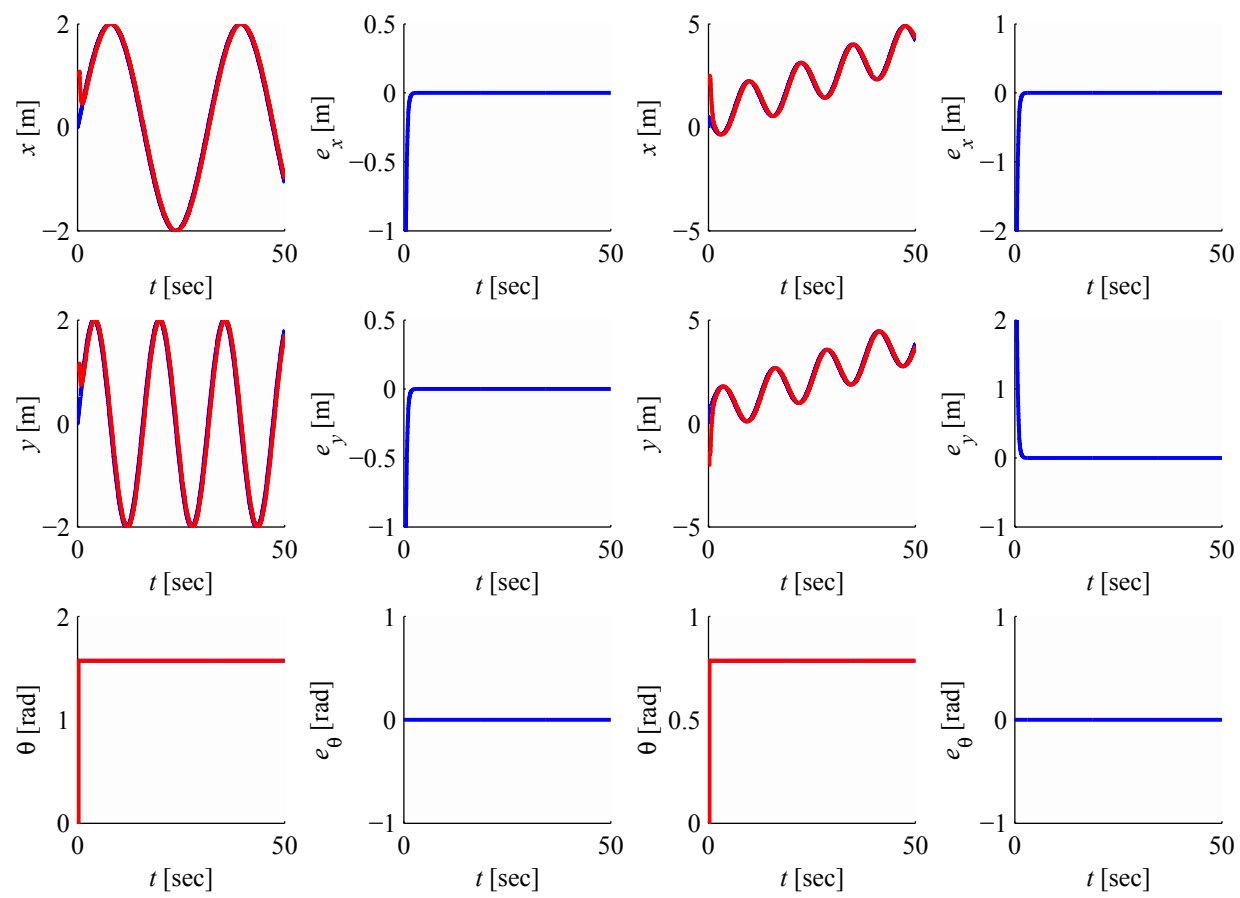

Fig. 12. Omnidirectional mobile robot states and errors. 
As with the unicycle-type mobile robot, the behavior of the omnidirectional mobile robot in the $X-Y$ plane (red) and its desired trajectory (blue) is depicted in Fig. 11 for both simulations. The small cross and circular markers denote the same elements as in Fig. 9. Once more, the mobile robot lags its desired trajectory due to the time-delay.

The mobile robot's states (red) compared to their reference (blue) together with their errors are shown in the different columns of Fig. 12 using the same distribution as in Fig. 10. Once more the state plots show a slight displacement between the reference trajectory and the states which is due to the time-delay and is barely noticeable since the simulation time to time-delay ratio is very high. The error plots once more converge or practically converge to zero, which means that the omnidirectional mobile robot is tracking its reference trajectory after a time $\tau$.

Remark 5.1 The proposed prediction structure has shown to be highly sensitive to a mismatch in the orientation's initial condition. This issue appears in both types of mobile robots and is the reason why the initial orientation in all the simulations was set to zero. In other words, if the models' and system's initial orientations differ, the system's performance is affected. Although not ideal, in a practical setting the robot's initial orientation can be determined beforehand in order to set the models' initial condition.

\section{Conclusions and Future Work}

A Smith-type predictor compensator has been proposed for two types of wheeled mobile robots subject to either an input or a bilateral time-delay. Using the exact discrete-time posture kinematic model of the mobile robots, the predictor's structure allows to implement noncausal control laws whose design is based on the delay free system. Numerical simulations show that the mobile robots will track a delayed version of the reference trajectory.

Many possible improvements to the proposed prediction-control scheme are possible. As noted by (Michiels and Niculescu, 2007), most of the work regarding the Smith predictor focuses on its robustness and its disturbance rejection characteristics. These issues are definitely relevant in the context of mobile robotics and should therefore be followed closely. For example, an adaptive algorithm that compensates for mismatch in the time-delay model would significantly improve the system's performance. A further extension of this work to robotic manipulators is also a possibility. In this case, issues such as the reflection of the contact and the driving forces has to be considered.

\section{References}

Brockett, R.W. (1983). Asymptotic stability and feedback stabilization. In Differential Geometric Control Theory, R.W. Brockett, R.S. Milman and H.J. Sussmann, Eds., Birkhäuser, Boston, pp. 181-191.

Canudas De Wit, C., Siciliano, B., Bastin, G., Brogliato, B., Campion, G., DAndrea-Novel, B., De Luca, A., Khalil, W., Lozano,R., Ortega, R., Samson, C. and Tomei, P. (1996). Theory of Robot Control. Springer-Verlag, London.

Campion, G. , Bastin, G. and DAndrea-Novel, B. (1996). Structural properties and classification of kinematic and dynamic models of wheeled mobile robots. IEEE Transactions on Robotics and Automation, 12(1), pp. 4762.

Henson, M. A. and Seborg, D. E.. (1994). Time delay compensation for nonlinear processes. Industrial Engineering Chem., 33(6):14931500. 
Hokayem, P.F. and Spong, M.W. (2006). Bilateral Teleoperation: An Historial Survey. Automatica, 42(12):2035-2057.

Jerome, N.F. and Ray, W.H. (1986). High-performance multivariable control strategies for systems having time delays. AIChE Journal, 32(6):914931.

Kotta, U. (1995). Inversion method in the discrete-time nonlinear control systems synthesis problems. Springer Berlin/Heidelberg, Berlin, Germany.

Kravaris, C. and Chung, C.B. (1987). Nonlinear feedback synthesis by global input/output linearization. AIChE Journal, 33(4):592-603.

Kravaris, C. (1988). Input/Output linearization: A nonlinear analog of placing poles at process zeros. AIChE Journal, 34(11):1803-1812.

Kravaris, C. and Wright, R. A. (1989). Deadtime compensation for nonlinear processes. AIChE Journal, 35(9):15351542.

Lefeber, E., Jakubiak, J., Tchoń, K. and Nijmeijer, H. (2001). Observer based kinematic tracking controllers for a unicycle-type mobile robot. In Proceedings of the 2001 IEEE International Conference on Robotics E Automation, Seoul, Korea.

Michiels, W. and Niculescu, S.I. (2007). Stability and Stabilization of Time-Delayt Systems: An Eigenvalue Based Approach. SIAM, Philadelphia.

Morari, M. and Zafiriou, E. (1989). Robust process control. Prentice Hall, New Jersey.

Nešić, D. and Loría, A. (2004). On uniform asymptotic stability of time-varying parameterized discrete-time cascades. IEEE Transactions on Automatic Control, 49(6):875-887.

Orosco-Guerrero, R., Velasco-Villa, M. and Aranda-Bricaire, E. (2004). Discrete-time controller for a wheeled mobile robot. In Proc. XI Latin-American Congress of Automatic Control, La Habana, Cuba.

Salgado-Jimnez, T. (2000). Design, implementation and control of a mobile robot. Masther's thesis, CINVESTAV IPN, Mexico.

Smith, O.J.M. (1957). Closer control of loops with dead time. Chem. Eng. Prog., 53, pp. 217-219. 


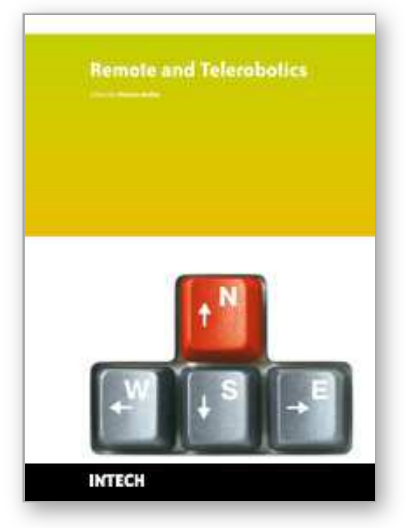

\author{
Remote and Telerobotics \\ Edited by Nicolas Mollet
}

ISBN 978-953-307-081-0

Hard cover, 220 pages

Publisher InTech

Published online 01, March, 2010

Published in print edition March, 2010

Any book which presents works about controlling distant robotics entities, namely the field of telerobotics, will propose advanced technics concerning time delay compensation, error handling, autonomous systems, secured and complex distant manipulations, etc. So does this new book, Remote and Telerobotics, which presents such state-of-the-art advanced solutions, allowing for instance to develop an open low-cost Robotics platform or to use very efficient prediction models to compensate latency. This edition is organized around eleven high-level chapters, presenting international research works coming from Japan, Korea, France, Italy, Spain, Greece and Netherlands.

\title{
How to reference
}

In order to correctly reference this scholarly work, feel free to copy and paste the following:

Alejandro Alvarez-Aguirre (2010). Predictor Based Control Strategy for Wheeled Mobile Robots Subject to Transport Delay, Remote and Telerobotics, Nicolas Mollet (Ed.), ISBN: 978-953-307-081-0, InTech, Available from: http://www.intechopen.com/books/remote-and-telerobotics/predictor-based-control-strategy-for-wheeledmobile-robots-subject-to-transport-delay

\section{INTECH}

open science | open minds

\section{InTech Europe}

University Campus STeP Ri

Slavka Krautzeka 83/A

51000 Rijeka, Croatia

Phone: +385 (51) 770447

Fax: +385 (51) 686166

www.intechopen.com

\section{InTech China}

Unit 405, Office Block, Hotel Equatorial Shanghai

No.65, Yan An Road (West), Shanghai, 200040, China

中国上海市延安西路65号上海国际贵都大饭店办公楼405单元

Phone: +86-21-62489820

Fax: +86-21-62489821 
(C) 2010 The Author(s). Licensee IntechOpen. This chapter is distributed under the terms of the Creative Commons Attribution-NonCommercialShareAlike-3.0 License, which permits use, distribution and reproduction for non-commercial purposes, provided the original is properly cited and derivative works building on this content are distributed under the same license. 